\title{
Un Trématode nouveau. \\ Parasite de Rana pipiens Schreber, 1872 \\ de la République de Costa Rica (Amérique centrale)
}

\author{
par Eduardo CABALLERO et C. Guillermina CABALLERO R. \\ [Facultad de Ciencias Biológicas, Universitad de Nuevo Leôn, México \\ Instituto de Biología, Universidad Nacional Autónoma de México]
}

\begin{abstract}
Résumé
Un nouveau Trématode, Brenesia chabaudi n. g., n. sp., recueilli dans l'intestin d'un Ranidae capturé à Costa Rica, Améi ique Centrale, Rana pipiens Schreber, 1872, est décrit et discuté.

Par la présence d'une vésicule excrétrice en « $V$ », de vitellogènes en position céphalique, de caecums très courts, ainsi que par la forme et la situation de l'appareil reproducteur, ce nouveau genre appartient à la famille Lecithodendriidae Odhner, 1911. Brenesia n. g. est très proche de deux genres: Pleurogenoides Travassos, 1921 et Pseudosonsinotrema Dollfus, 1951. Il diffère du premier de ces genres par la forme et la structure de la poche du cirre et du métraterme et par la situation de l'ovaire et des testicules; ces mêmes caractères le différencient de Pseudosonsinotrema. Chez notre espèce, le métraterme est entouré de cellules glandulaires, c'est le seul caractère qui la rapproche de Pseudosonsinotrema japonicum (Yamaguti, 1936) Manter et Pritchard, 1964, mais par les caractères déjà mentionnés, elle est différente de $P$. japonicum.
\end{abstract}

\section{Summary}

A new Trematode Brenesia chabaudi n. g., n. sp., from the intestin of a Ranidae collected in Costa Rica, Central America, Rana pipiens Schreber, 1872, is described and discussed. 
By the presence of an excretory vesicle V-shaped, vitellaria in cephalic position, short caeca, form and situation of reproductive system, this new genus belongs to the family Lecithodendriidae Odhner, 1911. The nearest genus are Pleurogenoides Travassos, 1921 and Pseudosonsinotrema Dollfus, 1951. Brenesia n. g., differs from the first of these genus by the form and structure of the cirrus pouch, the metraterm and situation of the ovary and testes; these same characters made it different from Pseudosonsinotrema. In our species, the metraterm is surrounded by glandular cells, this is the only character that approaches it to Pseudosonsinotrema japonicum (Yamaguti, 1936) Manter and Pritchard, 1964, but by the mentioned characters it is quite different from $P$. japonicum.

Le trématode qui fait l'objet de cette étude nous a été confié par Rodrigo Brenes qui l'a récolté de l'intestin grêle de Rana pipiens Schreber, 1872, le 18 juillet 1967. Les huit exemplaires trouvés ont été aplatis entre lamelles, fixés dans une solution de bichlorure de mercure et colorés avec le carmin de Grenacher. Nous avons employé le terpineol pour les éclaircir et la résine synthétique neutre pour le montage définitif. La description a été réalisée sur quatre exemplaires.

\section{BRENESIA CHABAUDI, n.g., n. sp.}

Le corps des parasites, ovale et petit, mesure $0.714-0.743 \mathrm{~mm}$ de long sur 0.400 $0.500 \mathrm{~mm}$ de large. La cuticule épaisse de $0.003 \mathrm{~mm}$ est armée de petites épines qui s'étendent sur tout le corps. Elles sont plus abondantes dans la moitié antérieure, et mesurent $0.007 \mathrm{~mm}$ de long sur $0.002 \mathrm{~mm}$ de large au niveau de leur naissance.

Les ventouses sont grandes, légèrement sphériques. La ventouse buccale, musculeuse, subterminale, mesure $0.109-0.126 \mathrm{~mm}$ de diamètre antéro-postérieur sur 0.116$0.116 \mathrm{~mm}$ de diamètre transversal. La ventouse ventrale, légèrement plus grande que la ventouse buccale est située dans la moitié postérieure du corps, en arrière de l'équateur. Sa région antérieure est tangente, à droite, au réceptacle séminal, et à gauche à la poche du cirre. Elle mesure $0.119-0.139 \mathrm{~mm}$ de diamètre antéro-postérieur sur 0.126-0.129 $\mathrm{mm}$ de diamètre transversal. Le rapport, ventouse ventrale/ventouse buccale est : $1: 1.09 \times 1: 1.08$ à $1: 1.1 \times 1: 1.1$.

$\mathrm{La}$ bouche ample, légèrement circulaire mesure $0.068-0.071 \mathrm{~mm}$ de diamètre antéro-postérieur sur $0.078-0.082 \mathrm{~mm}$ de diamètre transversal. Il n'y a pas de prépharynx. Le pharynx, petit, piriforme et musculeux, mesure 0.034-0.041 mm de diamètre antéro-postérieur sur 0.037-0.037 mm de diamètre transversal. L'œsophage, tube étroit à parois minces, est court, mesure 0.044-0.068 mm de long sur 0.014-0.017 mm de large; la bifurcation intestinale se trouve au niveau du métraterme, à 0.108 $0.204 \mathrm{~mm}$ du bord antérieur du corps. Les caecums sont très courts, étroits dans la partie antérieure et larges dans la postérieure, tangents au testicule droit, à l'ovaire et au métraterme, mesurant le droit $0.119-0.187 \mathrm{~mm}$ de long sur $0.037-0.037 \mathrm{~mm}$ de large, le gauche de $0.116-0.204 \mathrm{~mm}$ de long sur $0.020-0.034 \mathrm{~mm}$ de large. 


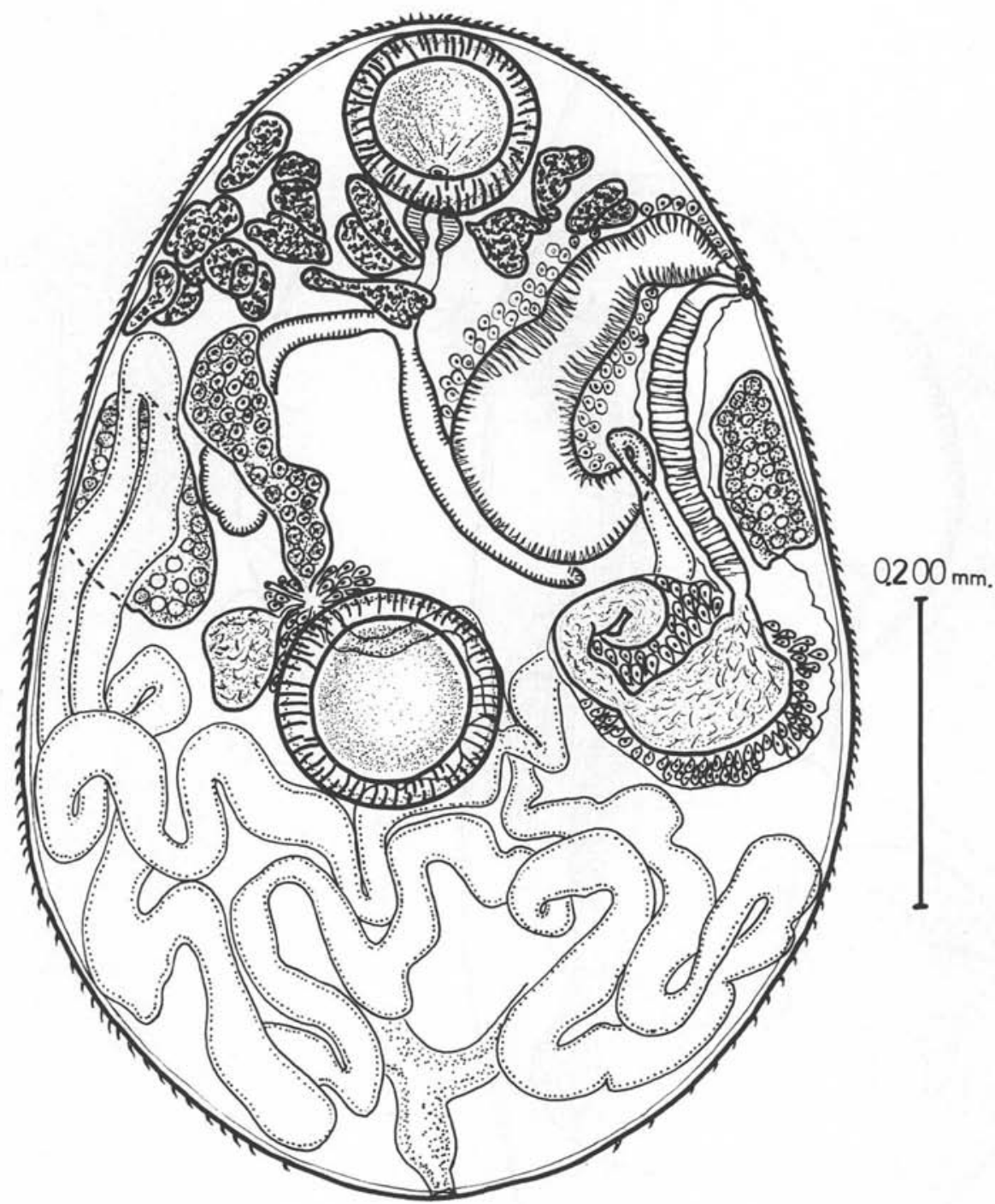

Fig. 1. - Brenesia chabaudi n. g. n. sp., préparation in toto. Vue ventrale

ApPAREIL GÉNITAL: L'orifice génital, situé antérieurement sur le bord latéral gauche du corps, à $0.187-0.221 \mathrm{~mm}$ du bord antérieur, mesure $0.014-0.014 \mathrm{~mm}$ de diamètre. Les testicules, petits, ovalés aux bords peu lobés, sont situés dans la moitié antérieure du corps, en avant de l'équateur ; le droit entre le caecum droit et la paroi du corps; le gauche dans l'aire limitée par, à gauche la paroi du corps, en avant le métraterme, en arrière la poche du cirre et, dorsalement la pars prostatique; le testicule 


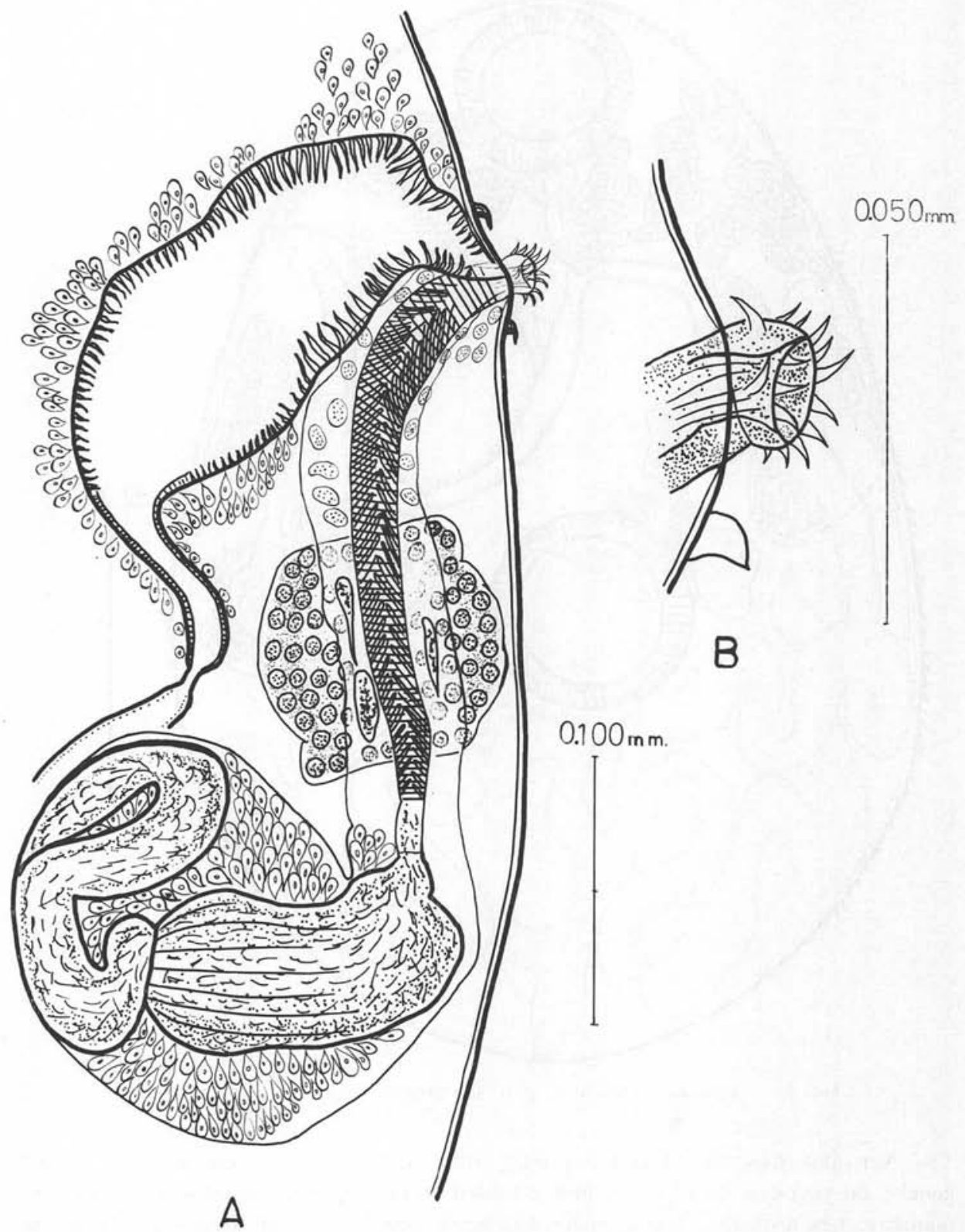

Fig. 2. - Brenesia chabaudi n. g. n. sp. : A) Détail de l'appareil reproducteur montrant un testicule, la poche du cirre, le métraterme et le cirre épineux sortant par l'orifice génital, situé sur le bord latéral gauche du corps. B) Détail du cirre épineux 
droit mesure $0.105-0.146 \mathrm{~mm}$ de long sur 0.075-0.095 $\mathrm{mm}$ de large, le testicule gauche, 0.085-0.109 $\mathrm{mm}$ de long sur 0.051-0.088 $\mathrm{mm}$ de large. La pocne du cirre est l'organe le plus développé de l'appareil reproducteur de ce trématode, elle possède la forme d'une «pipe », située en arrière de l'équateur du corps sur le côté gauche, entre la ventouse ventrale et le bord latéral. Elle est constituée de deux parties : une postérieure ovale et une autre antérieure tubuleuse (pars prostatique) qui suit parallèlement le bord latéral du corps et se termine dans l'orifice génital ; la partie postérieure mesure 0.109-0.119 $\mathrm{mm}$ de long sur 0.146-0.167 $\mathrm{mm}$ de large; l'antérieure (pars prostatique ?) mesure 0.214-0.238 $\mathrm{mm}$ de long sur $0.024-0.037 \mathrm{~mm}$ de large. La vésicule séminale est séparée par la présence de la glande prostatique ; la plus grosse partie occupe le fond de la poche, mesurant $0.075-0.112 \mathrm{~mm}$ de long sur $0.068-0.099 \mathrm{~mm}$ de large, la partie antérieure mesure $0.061-0.085 \mathrm{~mm}$ de long sur $0.051-0.102 \mathrm{~mm}$ de large. La glande prostatique bien développée, occupe la périphérie et le centre de la poche du cirre, entre les deux portions de la vésicule séminale; le cirre, corps cylindrique, petit et épineux mesure $0.014 \mathrm{~mm}$ de long sur $0.017 \mathrm{~mm}$ de large.

L'ovaire lobé, situé à droite de la ligne médiane, tangent ou couvrant partiellement le caecum intestinal du même côté, en avant de l'équateur du corps, allongé ou bien en forme de trèfie, mesure $0.099-0.160 \mathrm{~mm}$ de long sur $0.051-0.088 \mathrm{~mm}$ de large. L'ootype et la glande de Mehlis se trouvent en arrière de l'ovaire, proches de la ligne médiane équatoriale du corps. Nous avons bien observé le canal de Laurer et, le réceptacle séminal, petit, qui mesure 0.065-0.068 mm de long sur 0.041-0.068 $\mathrm{mm}$ de large. L'utérus, prend naissance dans la région de l'ootype et se dirige obliquement vers la ligne médiane équatoriale du corps, croise l'acétabulum en se dirigeant vers l'extrémité postérieure gauche du corps, par derrière ia poche du cirre, puis remonte vers la ventouse ventrale, dès son bord postéro-latéral droit traverse vers l'aire droite latéra'e du corps et s'y étend en remplissant toute l'aire comprise entre le bord postérieur du corps et le testicule droit en le dépassant, puis, l'utérus descend jusqu'au bord postérieur de la ventouse ventrale et obliquement remonte, tangent au bord interne de la poche du cirre, se mettant finalement en communication avec le métraterme. Le métraterme est un organe aussi grand que la poche du cirre, situé obliquement entre le caecum gauche et l'orifice génital, sa paroi externe est entourée de multiples cellules glandulaires; dans sa partie intérieure, constituant la paroi, il existe des cellules allongées, dirigées vers la cavité qui est ample dans la région postérieure de cet organe et étroite, comme un tube, dans la région antérieure. S'ouvrant dans l'orifice génital, cet organe, mesure 0.214$0.309 \mathrm{~mm}$ de long sur 0.075-0.078 $\mathrm{mm}$ de large. Les œufs nombreux, à coquille mince, jaunâtres, operculés, portant un petit mamelon à l'extrémité postérieure ; ils mesurent 0.019-0.019 $\mathrm{mm}$ de long sur 0.008-0.009 $\mathrm{mm}$ de large.

Les vitellogènes sont rares, grands, lobés, soit placés de chaque côté de la ventouse buccale, entre cet organe et le métraterme à gauche et, entre le testicule, le caecum et la ventouse buccale à droite, soit étendus de toute la région dorsale à la ventouse buccale, c'est-à-dire, que les vitellogènes occupent fondamentalement les aires antérieures dorsales et latérales à la ventouse buccale et le pharynx. 
Le pore excréteur, ample, s'ouvre dans l'extrémité postérieure du corps, il communique avec une vésicule excrétrice en $\mathrm{V}$.

HôTE: Rana pipiens Schreber, 1872.

LOCALISATION: Intestin grêle.

Localité : Puerto Viejo, Sarapiqui, Provincia de Heredia, Costa Rica, Amérique Centrale.

HoLOTYPE : Collection Helminthologique de E. Caballero y C., $\mathrm{n}^{\circ} 504$.

Paratypes: Collection Helminthologique de la Faculté de Microbiologie de l'Université de Costa Rica et de l'Institut de Biologie de l'Université Nationale Autonome de Mexico, $\mathrm{N}^{\circ} 225-6$.

BRENESIA n. gen.

Diagnosis: Lecithodendriidae. Corps petit, ovale couvert d'épines; ventouses grandes ; acetabulum plus grand que la ventouse buccale, situé dans la moitié postérieure du corps, en arrière de l'équateur; pharynx petit; œsophage court ; caecums très courts et sacciformes. Orifice génital sur le bord latéro-antérieur gauche du corps; testicules légèrement lobés, tangents au bord latéral de la moitié antérieure du corps ; poche du cirre située sur le côté gauche, équatoriale, formée de deux régions: une postérieure ovale et une autre antérieure tubuleuse (pars prostatique ?) s'ouvrant dans l'orifice génital; vésicule séminale interne divisée en deux par la présence d'une glande prostatique bien développée, des deux régions, la postérieure est grosse et l'autre, anterieure, petite, se termine dans la portion tubuleuse de la poche du cirre. Ovaire tangent ou couvrant le caecum droit, de forme lobée ou en trèfle; ootype, glande de Mehlis, canal de Laurer et réceptacle séminal présents, situés en arrière de l'ovaire ; utérus largement développé, occupant principalement la moitié postérieure et latérale droite du corps: métraterme grand, situé entre le caecum gauche et la portion antérieure de la poche du cirre, pourvu à l'intérieur d'une paroi épaisse de cellules allongées, orientées vers la lumière de l'organe; œufs nombreux, operculés, à coquille mince et pourvus dans leur extrémité postérieure d'un mamelon. Vitellogènes rares, gros, situés de part et d'autre de la ventouse buccale, sur la région dorsale de cet organe. Pore excréteur sur le bord postérieur du corps; vésicule excrétrice en V.

Espèce type: Brenesia chabaudi n. g., n. sp. Parasite de l'intestin grêle de Ranidae Bonaparte, 1831 (Amphibia Linnaeus, 1758), d’Amérique Centrale.

\section{Discussion}

Par la présence d'une vésicule excrétrice en $\mathrm{V}$, de vitellogènes dans la région latéro-céphalique, de caecums très courts, par la forme et la localisation de l'appareil reproducteur, ce nouveau genre appartient à la famille Lecithodendriidae Odhner, 1911. Il est très proche des genres Pleurogenoides Travassos, 1921 et Pseudosonsino- 
trema Dollfus, 1951. Il diffère du premier de ces genres, par la forme et structure de la poche du cirre, du métraterme et par la situation de l'ovaire et des testicules; ces mêmes caractères le font différent de Pseudosonsinotrema, bien que, chez l'espèce $P$. japonicum (Yamaguti, 1936), Manter et Pritchard, 1964, le métraterme est entouré d'une région glandulaire, la structure de cet organe et celle de la poche du cirre ainsi que leur position sont très différentes de cette espèce.

Par tous ces caractères, nous pensons donc que notre espèce est nouvelle et nous la nommons Brenesia chabaudi n. g. n. sp. Le nom du genre est dédié à $\mathbf{M}$. Rodrigo Brenes, M. de l'Université de Costa Rica et le nom de l'espèce à $\mathrm{M}$. le Professeur Alain G. Chabaud du Museum National d'Histoire Naturelle de Paris, qu'ils veuillent trouver ici notre reconnaissance pour leur labeur dans le domaine de la Parasitologie.

Nous remercions vivement le Professeur R. Ph. Dollfus, qui, lors d'un court séjour à Paris a mis à notre disposition l'espèce-type du genre Pseudosonsinotrema, pour la comparer avec l'espèce que nous venons de décrire, nous permettant ainsi de conclure aisément que nos spécimens constituent un nouveau genre.

\section{Bibliographie}

Balozet (L.) and Callot (J.), 1938. - Trématodes de Tunisie. 1. Trématodes de Rana ridibunda Pallas. Arch. Inst. Past. Tunisie, 27 (1) : 18-30.

Dollfus (R.-Ph.), 1951. - Miscellanea helminthologica marroccana. I. Quelques trématodes, cestodes et acanthocéphales. Arch. Inst. Past. Maroc, 4 (3) : 104-229.

Fischthal (F. H.) et Kuntz (R. E.), 1965. - Digenetic Trematodes of Amphibia and Reptiles from North Borneo (Malaysia). Proc. Helm. Soc. Washington, 32 (2) : 124-136.

Manter (H. W.) et Pritchard (M. H.), 1964. - 5. Vermes-Trematoda. Ann. Mus. Roy. Afr. Centr. Zool. (132) : 75-101.

Merra (H. R.), 1928. - Trematode parasites of the Pleurogenetinae from Rana tigrina with a Revision and Synopsis of the Subfamily. Allahabad Univ. Stud., 4: 63-118.

SKRJABIN (K. I.), 1948. - Semeystvo Lecithodendriidae Odhner, 1911. Trematody jivotnyj i cheloveka. Osnovy trematodologii, 2 : 337-590. Isdatelstvo Akademii Nauk. S.S.S.R. Moskva-Leningrad. En russe.

—, 1966. - Semeystvo Lecithodendriidae Odhner, 1911. Trematody jivotnyj i cheloveka. Osnovy trematodologii, 22 : 81-88. Isdatelstvo Akademii Nauk. S.S.S.R. Moskva. En russe.

Travassos (L.), 1930. - Pesquizas helminthologicas realizadas em Hamburgo. VI. Genero Pleurogenoides Travassos, 1921 (Trematoda : Lecithodendriidae). Mem. Inst. Oswaldo Cruz, 24 (2) : 63-71.

Yamaguti (S.), 1936. - Studies on the helminth Fauna of Japan. Part. 14. Amphibian Trematodes. Jap. Jour. Zool., 6 (4) : 551-576.

—, 1958. - Systema Helminthum. The Digenetic Trematodes of Vertebrates, 1 (1-2) : XI + 1575. Interscience Publishers, New York-London. 